\title{
Turbulence Evolution and Shock Acceleration of Solar Energetic Particles
}

\author{
Chee $\mathrm{K} . \mathrm{Ng}$ \\ Code 661, NASA Goddard Space Flight Center, USA \\ Department of Astronomy, University of Maryland, College Park, USA
}

\begin{abstract}
We model the effects of self-excitation/damping and shock transmission of Alfvén waves on solar-energetic-particle (SEP) acceleration at a coronal-mass-ejection (CME) driven parallel shock. SEP-excited outward upstream waves speedily bootstrap acceleration. Shock transmission further raises the SEP-excited wave intensities at high wavenumbers but lowers them at low wavenumbers through wavenumber shift. Downstream, SEP excitation of inward waves and damping of outward waves tend to slow acceleration. Nevertheless, $>2000 \mathrm{~km} / \mathrm{s}$ parallel shocks at $\sim 3.5$ solar radii can accelerate SEPs to $100 \mathrm{MeV}$ in $<5$ minutes.
\end{abstract}

Keywords: coronal shock acceleration, solar energetic particles, self-excited plasma wave PACS: $96.50 . \mathrm{Vg}$, 96.50.Fm, 96.50.Tf, 96.50.Pw

\section{INTRODUCTION}

Self-excited Alfvén wave turbulence near CME-driven shocks enhance SEP scattering and first-order Fermi acceleration [1, 2]. Steady-state models [e.g. 3, 4], which do not address acceleration time scale, consider only self-excitation of upstream (US) waves, because the constant-density SEPs downstream (DS) are purely convected. Timedependent models, however, must treat DS wave evolution as well, because the SEPs stream down density gradients in both directions away from shock. For simplicity we consider energetic protons interacting resonantly with circularly polarized parallel and anti-parallel Alfvén wave modes $\left(\mathrm{R}^{ \pm}, \mathrm{L}^{ \pm}\right)$near a parallel shock and focus on two processes affecting wave evolution: SEP excitation/damping and shock transmission.

We find that US, protons accelerated up to energy $E$ amplify outward waves and damp inward waves down to some minimum wavenumbers $k$. Each SEP-amplified US Alfvén wave mode then crosses the shock to emerge DS as a small inward mode plus a dominant outward mode, amplified and shifted to higher $k$ by shock transmission, consistent with $[5,6,7,8]$. DS, first, the $k$-shifted waves resonate with lower-energy protons. Secondly, SEPs scattered back to the shock by inward waves lose energy compared to those scattered by outward waves. Thirdly, the inward streaming SEPs damp outward waves and amplify inward waves. ${ }^{1}$ These DS processes slow acceleration and soften the SEP energy spectrum at high energy, consistent with the finding in a simpler model by [10]. We present model calculations below to quantify these effects and the influence of the outer boundary condition.

\footnotetext{
${ }^{1}$ Inward/outward relative to the plasma. All modes travel inward everywhere relative to the shock.
} 


\section{MODEL}

We adopt a radial mean magnetic field $B=B_{0}\left(r_{0} / r\right)^{2}$ and plasma proton number density $n_{\mathrm{H}}=n_{\mathrm{H}, 0}\left(r_{0} / r\right)^{2}$, hence Alfvén speed $V_{\mathrm{A}}=V_{\mathrm{A}, 0}\left(r_{0} / r\right)$, with $r$ the radial distance. $B_{0}=0.143$ gauss, $n_{\mathrm{H}, 0}=2 \times 10^{5} \mathrm{~cm}^{-3}$, and $V_{\mathrm{A}, 0}=700 \mathrm{~km} \mathrm{~s}^{-1}$ are typical values at $r_{0}=3.5 r_{\odot}[9,11]$. Relative to the Sun, the shock, US, and DS plasma travel at $V_{\mathrm{sh}}=$ $2500 \mathrm{~km} \mathrm{~s}^{-1}, V_{\mathrm{u}}=83 \mathrm{~km} \mathrm{~s}^{-1}$, and $V_{\mathrm{d}}=1880 \mathrm{~km} \mathrm{~s}^{-1}$, respectively. At time $t=0$, the shock is at $r_{\mathrm{sh}}=r_{0}$, fluid compression ratio $c_{f}=3.895$, and Mach number $M_{A}=3.45$. The outward and inward Alfvén wave velocities are $W_{ \pm}(r, t)=V(r, t) \pm V_{\mathrm{A}}(r, t)$. The SEP and Alfvén wave distributions evolve according to

$$
\begin{gathered}
\partial_{t} F+\partial_{r}\left[\left(\mu v+W_{+}\right) F\right]+\partial_{\mu}(\dot{\mu} F)+P \partial_{P}(F \dot{P} / P)= \\
\partial_{\mu}\left(D_{\mu \mu} \partial_{\mu} F+D_{\mu P} \partial_{P} F\right)+P^{-2} \partial_{P}\left[P^{2}\left(D_{P \mu} \partial_{\mu} F+D_{P P} \partial_{P} F\right)\right] \\
\dot{\mu}=\left(v+\mu W_{+}\right)\left(1-\mu^{2}\right) / r-\left[\left(1-\mu^{2}\right) / v\right]\left[\partial_{t} W_{+}+\left(\mu \nu+W_{+}\right) \partial_{r} W_{+}\right] \\
\dot{P} / P=-W_{+}\left(1-\mu^{2}\right) / r-(\mu / v)\left[\partial_{t} W_{+}+\left(\mu v+W_{+}\right) \partial_{r} W_{+}\right] \\
\partial_{t} \Psi_{\sigma}+\partial_{r}\left(W_{\sigma} \Psi_{\sigma}\right)+\eta \partial_{\eta}\left[\left(2 W_{\sigma} / r-d W_{\sigma} / d r\right) \Psi_{\sigma}\right]=\gamma_{\sigma} \Psi_{\sigma} \\
D_{\mu \mu}=(v / 2 P)^{2} \sum_{\sigma} d k I^{\sigma} R_{\mu \mu}^{\sigma}, \\
\gamma_{\sigma}(k, r, t)=2 \pi^{2} c e^{3} g_{\sigma} \dot{V}_{\mathrm{A}} \iint d \mu d P P^{3} R_{\mu \mu}^{\sigma} G f /\left[\mathscr{E}^{2}\left(1-\mu V_{\sigma} / v\right)^{2}\right] .
\end{gathered}
$$

In the above, $F(P, \mu, r, t)=f(P, \mu, r, t) B_{0} P /\left(B P_{0}^{3}\right)$, with $f$ the SEP phase-space density in mixed coordinates: $r, t$ in inertial frame; velocity $v$, rigidity $P$, and pitch cosine $\mu$ in local outward wave frame. $P_{0}=$ a constant. $D_{\alpha \beta}=$ momentum diffusion tensor [12]. Besides focusing and adiabatic deceleration, $\dot{\mu}$ and $\dot{P}$ in eqs. (2) and (3) include differential frame transformation of $(\mu, P)$ following particles. $I^{\sigma}(k, r, t)=$ magnetic intensity of $\sigma$-mode Alfvén wave. $\Psi_{\sigma}(\eta, r, t) \equiv 2 I^{\sigma} \eta\left|W_{\sigma}\right| /\left(\eta_{0} V_{\mathrm{A}}\right) \propto$ wave action density, $\eta=k / B, \eta_{0}=$ a constant, and $\gamma_{\sigma}=$ fractional growth rate of $I^{\sigma}$. In eq. (6), $\mathscr{E}=$ total particle energy; $G f \equiv \partial_{\mu} f-\left(\mu \partial_{\mu} f-P \partial_{P} f\right) V_{\sigma} / v ; R_{\mu \mu}^{\sigma}\left(\mu, P, k, V_{\mathrm{A}}, B\right)=$ wave-particle resonance function [12]; $g_{\sigma}=1, V_{\sigma}=0$ for $\sigma=R+, L+$; and $g_{\sigma}=-1, V_{\sigma}=-2 V_{\mathrm{A}}$ for $\sigma=R-, L-$.

US, $I^{R+}=I^{L+} \propto k^{-5 / 3}$ are initialized to a steady-state solution of the wave kinetic equation (4) and $I^{R-}=I^{L-}=0.04 I^{R+}$; these are normalized to give mean free path $\lambda>0.4 \mathrm{AU}$ at $1 \mathrm{MeV}$ [12]. DS, $I^{\sigma}$ and $n_{\mathrm{H}}$ are derived via shock transition. At $t=0$, the DS shock-transmitted waves are already enhanced by a factor of $\approx 80$. We specify US and DS isotropic seed protons $f_{\text {seed }}=5 b n_{\mathrm{H}}\left(r_{\mathrm{sh}}\right) P^{-8} /\left[4 \pi\left(P_{a}^{-5}-P_{b}^{-5}\right)\right],\left(P_{a}<P<P_{b}\right)$, with $b=0.0028, P_{a}=5.92 \mathrm{MV}(19 \mathrm{keV})$, and $P_{b}=9.95 \mathrm{MV}(53 \mathrm{keV})$.

Eqs. (1) and (4) are recast in $x$ where $x=r-r_{\mathrm{sh}}(t)$ (hence $n_{\mathrm{H}}, V_{\mathrm{A}}$, etc. become timedependent) and solved numerically via finite difference approximation using operator splitting and dynamic time steps [12] in a box comoving with the shock. There are 200 DS cells and 400 or 2000 US cells. The grid is defined as follows: $x_{j}=x_{j}+j \Delta x, j=$ $-200: 400$ or $2000, \Delta x=2.14 \times 10^{-4} r_{\odot} ; \mu_{i}=(i+1 / 2) \Delta \mu, i=-20: 19, \Delta \mu=0.05 ; \ln P_{\ell}=$ 
$\ln P_{1}+(\ell-1) \Delta \ln P, \ell=1: 46, \Delta \ln P=0.08664, P_{1}=10.38 \mathrm{MV} ;$ and $\log \eta_{m}=\log \eta_{1}+$ $(m-1) \Delta \log \eta) m=1: 48, \Delta \log \eta=0.05, \log \eta_{1}=4.87 \times 10^{-4} \mathrm{MV}^{-1}$.

At the moving inner and outer boundaries, we specify $F_{i, \ell, j=-201}=F_{i, \ell, j=-200}$, $F_{i, \ell, j=401}=F_{i, \ell, j=400}, \Psi_{\sigma, m, j=401}=\Psi_{\text {ambient }}$. Protons are accelerated via $P$-transport in cells adjacent to the shock (eq. (3)), where $\left|\mu \Delta W_{+} / \nu_{\ell}\right|<0.2 \Delta \ln P$; otherwise, frame transformation is performed on shock-crossing protons to evaluate more accurately the $\mu$ and $P$ increments. Each US wave mode $I_{u}\left(k_{u}\right)$ crossing the shock is converted preserving helicity into two DS modes $I_{d}\left(k_{d}\right)$ as follows: $[5,6]$.

$$
\begin{aligned}
I_{d}\left(k_{d}\right) / I_{u}\left(k_{u}\right) & =\left(1+\zeta_{u} \zeta_{d} / \sqrt{c_{f}}\right)^{2} c_{w} / 4, \\
c_{w} & =c_{f}\left(M_{A}-\zeta_{u}\right) /\left(M_{A}-\zeta_{d} / \sqrt{c_{f}}\right)
\end{aligned}
$$

$c_{w} \equiv k_{d} / k_{u} \equiv$ wave compression ratio, $\zeta_{u}= \pm 1$ and $\zeta_{d}= \pm 1$ for outward/inward US and DS waves. Since nonlinear processes limit wave growth, we impose $I^{\sigma} \leq I_{\text {sat }}=$ $B^{2} /(3 \pi k)$ so that $\lambda \geq 3 r_{g}, r_{g} \equiv$ gyroradius, to stay within quasilinear theory.

\section{RESULTS}

Figures $1 \mathrm{a}$ and $1 \mathrm{~b}$ show the coupled evolution of the proton intensity $j_{E}$ versus $E$ and the Alfvén wave intensity $I^{R+}$ versus $k / B$, respectively, just US of the shock in the reference case. In 5 minutes protons are accelerated to $100 \mathrm{MeV}$ and wave growth spreads from $k / B \approx 0.3 \mathrm{MV}^{-1}$ to $0.002 \mathrm{MV}^{-1} .20-50 \mathrm{keV}$ seed protons emerge across the shock anisotropically and excite ambient $R+$ and $L+$ Alfvén waves at resonant wavenumbers $k \sim B /\left[P\left(\mu-V_{\sigma} / v\right)\right]$. The amplified waves enhance scattering, providing a positive feedback loop that rapidly bootstraps acceleration and wave growth. The strong wave growth results in the wave spectra being limited by $I_{\text {sat }}$.

Snapshots of the radial profiles of $j_{E}$ and $I^{R+}$ are shown in Figs. 1c, $1 \mathrm{~d}$ for $1.3 \mathrm{MeV}$ protons and 1e, if for $68 \mathrm{MeV}$ protons, respectively. At $1.3 \mathrm{MeV}$, proton acceleration "ignites" at $\sim 18 \mathrm{~s}$ and is in full throttle by $120 \mathrm{~s}$. Simultaneously, the US $\lambda$ falls from $0.4 \mathrm{AU}$ to $\sim 3 \times 10^{-7} \mathrm{AU}$ (Figs. $1 \mathrm{c}$ and $1 \mathrm{~d}$ ). In contrast, acceleration at $68 \mathrm{MeV}$ ignites later at $\sim 150 \mathrm{~s}$, when $\lambda$ falls below $1 \times 10^{-4}$ AU (Fig. 1f), and does not reach full throttle at $300 \mathrm{~s}$ (Fig. 1e).

TABLE 1. Model Runs

\begin{tabular}{lcccc}
\hline $\begin{array}{c}\text { Upstream } \\
\text { cells }\end{array}$ & $\begin{array}{c}\mathbf{D}_{\mu \mathbf{P}}, \mathbf{D}_{\mathbf{P P}} \\
\neq \mathbf{0} \boldsymbol{?}\end{array}$ & $\begin{array}{c}\text { Wave intensity } \\
\text { ratios preserved? }\end{array}$ & $\psi_{\mathbf{J L}+\mathbf{1}}=$ \\
\hline 1 & 400 & yes & no & ambient \\
2 & 400 & yes & Yes & ambient \\
3 & 400 & no & Yes & ambient \\
4 & 2000 & yes & Yes & $\left(\psi_{J L}+\right.$ ambient $) / 2$ \\
$5^{*}$ & 2000 & yes & Yes & ambient \\
6 & 400 & yes & Yes & $\psi_{J L}$ \\
\hline
\end{tabular}

${ }^{*}$ reference case 

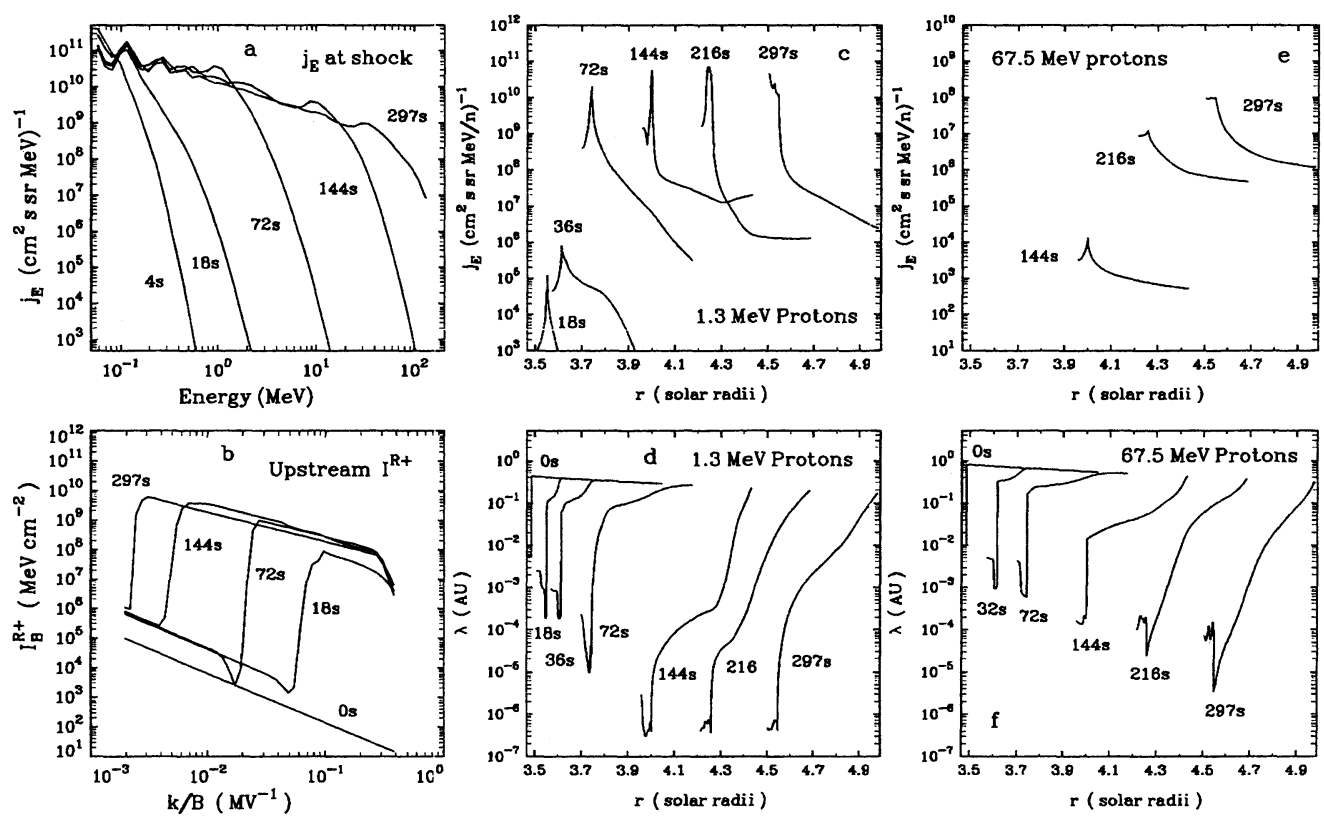

FIGURE 1. Evolution of: (a) $j_{E}$ vs $E$ proton intensity spectrum and (b) $I^{R+}$ vs $k / B$ Alfvén wave spectrum at the first US cell; (c) $j_{E}$ vs $r$ profile and (d) mean-free-path $\lambda$ vs $r$ profile of $1.3 \mathrm{MeV}$ protons. Panels (e) and (f) are as in (c) and (d) but for $67.5 \mathrm{MeV}$ protons.

The evolution of $I^{R+}$ and $I^{L-}$ DS is illustrated in Figs. $2 \mathrm{a}$ and $2 \mathrm{~b}$, respectively. These wave intensities have been enhanced by the shock transmission process and subject to wave saturation while maintaining the ratio of $I^{L-}(k) / I^{R+}(k)$ obtained from (eq. (7)). Both DS wave spectra are shifted to higher $k$ out of resonance with the currently highest energy protons except at small $\mu$. Furthermore, the $I^{R+}$ spectrum is shifted more than the $I^{L-}$ spectrum, so that $I^{L-}$ dominate at low $k$. Consequently the wave compression ratio experienced by the currently highest energy protons is weaker than that felt by lower energy protons.

For the reference case, we have employed 200 DS cells and 2000 US cells, ambient wave intensities at the moving outer boundary, and preserve the ratios of inward to outward wave intensities in applying ad hoc wave saturation. The consequences of varying these parameters or procedures on the SEP $j_{E}$ versus $E$ spectrum at $t=297 \mathrm{~s}$ are shown in Fig. 2c. The six model runs for comparison are summarized in Table 1 and spectrum 5 is for the reference case above.

Reducing the number of US cells from 2000 to 400 alone produces spectrum 2, which shows significant acceleration to only $30 \mathrm{MeV}$. This can be understood as follows. The SEPs amplify the $R+, L+$ ambient waves as they travel from the outer boundary to the shock. The proton intensity scale length is much longer at $>30 \mathrm{MeV}$ than at $<5 \mathrm{MeV}$ (Figs. 1c and 1e). Hence, for $<5 \mathrm{MeV}$ protons the resonant wave growth occurs mostly near the shock, but for $>30 \mathrm{MeV}$ protons, significant wave growth occurs beyond 400 cells. Therefore reduction of the US region has little effect on SEP acceleration 

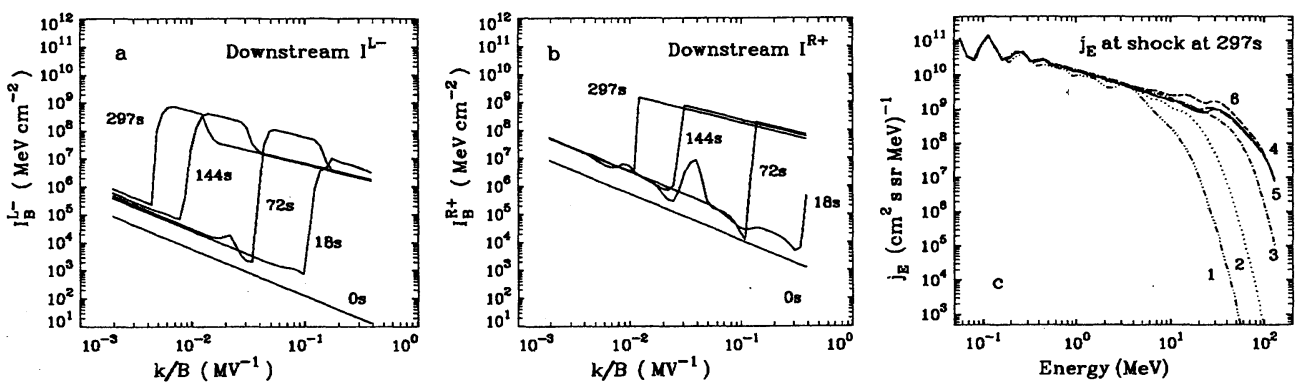

FIGURE 2. (a) DS $I^{L-}$, (b) DS $I^{R+}$, (c) $j_{E}$ shock spectra at $t=297 \mathrm{~s}$ in various simulations (Table 1)

at $<5 \mathrm{MeV}$ but significantly curtails it at $>30 \mathrm{MeV}$. Interestingly, accounting crudely for wave growth beyond cell 400 using the boundary condition $\left.\Psi\right|_{j=401}=\Psi_{j=400}$ yields spectrum 6, which slightly exceeds the reference spectrum 5 at high energies.

Setting $D_{\mu P}=D_{P \mu}=D_{P P}=0$ thereby turning off energy loss due to scattering by DS inward waves results in spectrum 3. Comparison of spectra 2 and 3 show that scattering by DS inward waves reduces SEP acceleration by $\sim 30 \mathrm{MeV}$. Spectrum 1 results from simply imposing wave saturation without maintaining the ratio of inward to outward DS wave intensities predicted by shock transmission. The consequent dominant DS $R$ - and $L$ - waves return particles to the shock with energy loss thereby slowing acceleration. Finally, spectrum 4 is produced with 2000 US cells and the modified outer boundary condition $\left.\Psi\right|_{j=2001}=\left(\left.\Psi\right|_{j=2000}+\Psi_{\text {ambient }}\right) / 2$. Spectra 4 and 5 are almost indistinguishable.

\section{DISCUSSION AND SUMMARY}

Shock transmission has subtly different implications on steady-state and time-dependent shock acceleration. Since $c_{w}>c_{f}$, steady-state test-particle theory immediately gives harder than classical SEP spectral index [6]. In our time-dependent shock acceleration model, US SEPs at $E<E_{\max }$ amplify outward Alfvén waves at $k>k_{\min }$. The amplified US waves are then shock-transmitted DS with $k_{\min }$ increased more for the outward than the inward daughter mode, resulting in (a) loss of resonance with the highest energy SEPs except at small $\mu$ and (b) a weaker $c_{w}$ compression at high energy with the DS inward mode. This situation is compounded by the SEPs damping (amplifying) DS outward (inward) waves, since the DS inward waves return SEPs to the shock with energy loss compared to outward waves. Acceleration and wave growth still proceed quickly, however, because high-energy SEPs of smaller $\mu$ are scattered by these waves. These processes are studied via the comparison runs reported above.

It is important to have $\mu$-dependence in the wave-particle resonance condition [12]. Quasilinear theory [13] gives $B / k \sim P\left(\mu-V_{\mathrm{A}} / v\right)$, so that waves excited by, e.g., 0.5 $\mathrm{MeV}$ protons at $\mu \sim 0.8$ can scatter $2 \mathrm{MeV}$ protons at $\mu \sim 0.4$. This coupling of low and high energy protons via the same resonant waves is essential for bootstrap shock acceleration and wave growth. If the scattering rate uses the popular "sharpened" 
resonance $B / k=P$ and the wave growth rate uses the correct $\mu$-dependence [e.g., 14], wave growth does not produce commensurate enhancement in the scattering rate for the most energetic SEPs and acceleration is slow. Going one step further in the sharpened resonance approximation and evaluating the wave growth rate from particles at a single energy, much faster acceleration is obtained [15]. However, the pair of wave growth and particle scattering rates, based on one-on-one relation between wavenumber and particle energy regardless of pitch angle, represents a fairly drastic approximation to the original quasilinear expressions. The sharpened resonance prohibits coupling between SEPs of different energies via wave interaction, so that in a time-dependent model, the few high energy SEPs must amplify their own resonant ambient waves before they experience enhanced scattering, slowing down acceleration.

Self-amplification of upstream Alfvén wave turbulence is a powerful process that rapidly bootstraps shock acceleration of SEPs. There is likely to be more scattering than imposed by the ad hoc $\lambda>3 r_{g}$ limit in our model [16]. Hence a $>2000 \mathrm{~km} / \mathrm{s}$ coronal shocks is likely to accelerate protons to $100 \mathrm{MeV}$ in $<5$ minutes.

The present model does not consider compressive wave modes, wave-wave interaction, nonlinear cascading, wave dissipation, shock obliquity, or the evolution of the shock structure by the SEPs and waves. Extending the model to include any of these poses a significant challenge. In focusing on the self-consistent interaction between SEPs and Alfvén waves, the present work has identified the importance of the interplay between SEP-Alfvén-wave interaction, shock transmission, and shock acceleration, in particular, the role of the downstream waves.

\section{ACKNOWLEDGMENTS}

I thank D. V. Reames, A. J. Tylka, and T. T. von Rosenvinge for valuable discussions. This work was supported by NASA LWS04-0000-0076 and SHP04-0016-0024.

\section{REFERENCES}

1. D. V. Reames, Space Sci. Rev., 90, 413 (1999)

2. M. A. Lee, Astrophys. J. Supp., 158, 38-67 (2005)

3. A. R. Bell, Mon. Not. R. Astron. S., 182, 147-156 (1978)

4. B. E. Gordon, M. A. Lee, E. Möbius, and K. J. Trattner, J. Geophys. Res., 104, 28263 (1999)

5. J. F. McKenzie and K. O. Westphal, Plant. Space. Sci, 17, 1029-1037 (1969).

6. R. Vainio and R. Schlickeiser, Astron. Astrophy., 343, 303-311 (1999)

7. G. M. Webb, A. Zakharian, M. Brio, and G. P. Zank, J. Plasma Phys., 61, 295 (1999)

8. A. Zakharian, G. M. Webb, M. Brio, and G. P. Zank, Proc 26th ICRC, Salt Lake City, Utah, 6, 351-354 (1999)

9. G. A. Dulk and D. J. McLean, Sol. Phys., 57, 279-295 (1978)

10. R. Vainio, Proc 27th ICRC, Hamburg, 6, 2054-2057 (2001)

11. M. Guhathakurta, E. C. Sittler, Jr., and D. McComas, Space Sci. Rev., 87, 199-206 (1999).

12. C. K. Ng, D. V. Reames, and A. J. Tylka, Astrophys. J., 591, 461-485 (2003).

13. J. R. Jokipii, Astrophys. J., 146, 480 (1966)

14. E. G. Berezhko, S. I. Petukhov, and S. N. Taneev, Astron. Letters 24, 122-130 (1998).

15. R. Vainio and T. Laitinen Astrophys. J., 658, 622-630 (2007).

16. G. P. Zank, G. Li, V. Florinski, H. Qiang, Lario, D., and C. W. Smith, J. Geophys. Res., 111, A06108 (2006) 